\title{
Longitudinally extensive transverse myelitis with AQP4 antibodies revealing ovarian teratoma ${ }^{\text {负 }}$
}

\author{
Marina Frasquet ${ }^{\text {a }}$, Luis Bataller ${ }^{\text {a,b,d,* }}$, Estefanía Torres-Vega ${ }^{\text {b,d }}$, María Durán-Moreno ${ }^{\mathrm{c}, \mathrm{d}}$,

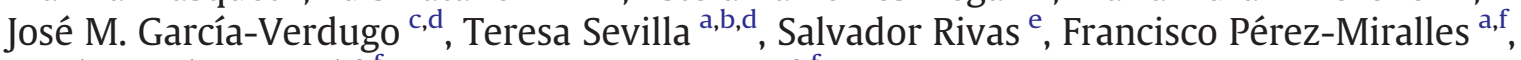 \\ María Simó-Castelló a,f, Bonaventura Casanova a,f \\ a Department of Neurology, Hospital Universitari i Politècnic La Fe, Valencia, Spain \\ ${ }^{\mathrm{b}}$ Laboratory of Neurology, Hospital Universitari I Politècnic La Fe, Valencia, Spain \\ c Department of Comparative Neurobiology, Cavanilles Institute of Biodiversity and Evolutive Biology, University of Valencia, Valencia, Spain \\ d Centro de Investigación Biomédica en Red sobre Enfermedades Neurodegenerativas, Valencia, Spain \\ e Department of Pathology, Hospital Universitari I Politècnic La Fe, Valencia, Spain \\ f Laboratory of Neuroimmunology, Hospital Universitari I Politècnic La Fe, Valencia, Spain
}

\section{A R T I C L E I N F O}

\section{Article history:}

Received 28 May 2013

Received in revised form 29 June 2013

Accepted 3 July 2013

Available online $\mathrm{xxxx}$

\section{Keywords:}

Transverse myelitis

Paraneoplastic syndrome

Autoimmune diseases

\begin{abstract}
A B S T R A C T
Paraneoplastic myelitis is a rare inflammatory disorder most frequently associated with solid tumors or lymphoproliferative disorders. Patients often harbor onconeuronal antibodies and their prognosis is usually poor. Here we report a 42-year old woman with longitudinally extensive transverse myelitis and aquaporin-4 (AQP4) antibodies that led to the diagnosis of ovarian teratoma. After tumor removal and immune therapy (including corticosteroids, plasma exchange, intravenous immunoglobulins and rituximab) the patient progressively improved achieving complete recovery. Histological study of the teratoma demonstrated neural tissue containing AQP4 expressing cells and intense inflammatory infiltrates, providing evidence for a possible paraneoplastic link between both disorders.
\end{abstract}

(C) 2013 Elsevier B.V. All rights reserved.

\section{Introduction}

Paraneoplastic myelitis is a rare complication of systemic cancer with poor prognosis. Most frequently associated neoplasms are solid tumors (breast, lung) and lymphoproliferative disorders. Clinical, radiological and pathological characteristics of paraneoplastic myelitis are diverse, and may include transverse inflammatory myelitis and necrotizing myelopathy (Darnell and Posner, 2011; Flanagan et al., 2011). Well-characterized onconeuronal antibodies assist in confirming the diagnosis. Anti-aquaporin-4 (AQP4) antibodies are detected in patients with neuromyelitis optica (NMO), a non-paraneoplastic relapsing inflammatory disorder affecting the spinal cord, optic nerves and sometimes the brain (Lennon et al., 2005). Of interest, a few patients with NMO-spectrum disorder with anti-AQP4 antibodies and systemic cancer have recently been reported (Mueller et al., 2008; Pittock and Lennon, 2008; Armağan et al., 2012). Here we report a patient with AQP4 antibody positive longitudinally extensive transverse myelitis (LETM) revealing ovarian teratoma. The teratoma expressed the AQP4 antigen, providing a possible paraneoplastic link between both diseases.

\footnotetext{
Study funding: Supported by FIS PI 12/01031.

* Corresponding author at: Department of Neurology, Hospital Universitari i Politècnic La Fe, Boulevar Sur s/n. 46026 Valencia, Spain. Tel.: + 34 961244000x485626.

E-mail address: bataller_lui@gva.es (L. Bataller).
}

\section{Case report}

A 42 year-old female presented to the emergency room with urinary retention. Transvaginal ultrasound demonstrated a right ovarian tumor which was confirmed on $\mathrm{CT}$ and demonstrated a heterogeneous cystic tumor with fat attenuation. Two days later a right adnexectomy was performed. Over the following $48 \mathrm{~h}$, the patient developed severe weakness in both legs. A neurology consultation was undertaken. She took no medications and smoked 20 cigarettes daily until the age of 34 . At the age of 12 she had a one-week episode of cheek paresthesia and blurred vision in one eye that improved spontaneously. She has a daughter diagnosed with rheumatoid arthritis. There were no recent symptoms of infection or vaccinations. Three weeks before admission she started noticing hiccups, nausea and vomiting, followed by burning pain with paresthesia in the upper chest, and problems with micturition that eventually led to urinary retention. Examination revealed normal cranial nerves. Strength, tone and reflexes were normal in the upper extremities. Both legs were severely paretic (2/5 Medical Research Council Scale), with flaccid tone and absent deep tendon reflexes. Cutaneoplantar responses were absent. There was a C2 sensory level for pain and light touch. Vibration and joint position sensations were normally perceived. The rest of the general and neurological examination was unremarkable. Spinal magnetic resonance imaging (MRI) is shown in Fig. 1. Brain MRI was unremarkable. Visual evoked potentials were normal. Routine laboratory investigations were unrevealing. Autoimmunity serological 

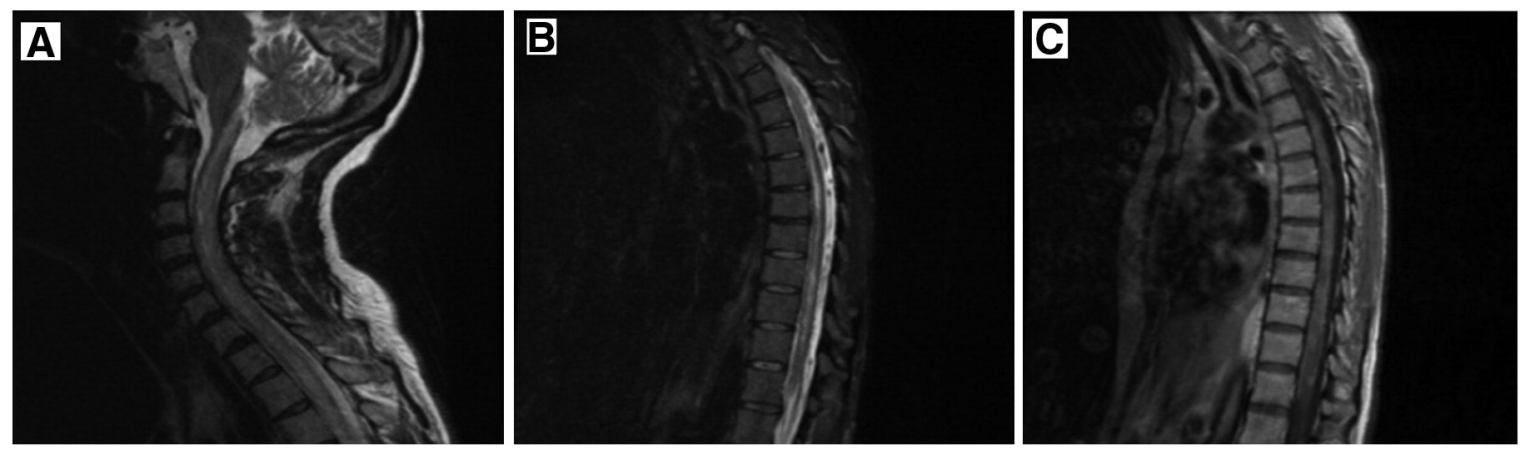

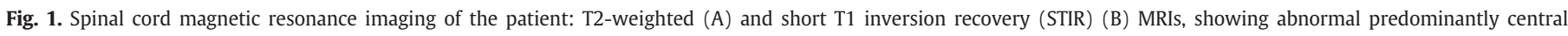

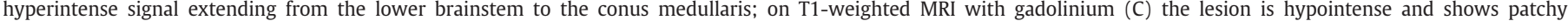
non-symmetric areas of mild contrast enhancement.

tests were negative, including anti-thyroid, anti-nuclear and anti-neutrophil cytoplasm antibodies, and serum immunofixation. Serologies for the following viruses were negative: CMV, EBV, VZV, HSV, HCV, HIV, HTLV 1 and 2. There was a positive HBV serology indicative of past infection (positive anti-HBs and anti-HBc antibodies, negative HBsAg). Treponema pallidum (TPHA and RPR) serology was negative. Alpha-fetoprotein levels were within normal limits. Cerebrospinal fluid analysis disclosed 260 white blood cells/mL (90\% mononuclear, $10 \%$ polymorphonuclear), mild increase in proteins $(80.5 \mathrm{mg} / \mathrm{dL})$ and normal glucose. CSF contained oligoclonal IgG and IgM bands. Microbiological CSF studies were negative, including conventional and mycobacterial cultures, and PCRs for JCV, HSV, VZV, EBV and CMV. CSF cytology was negative and flow cytometry analysis revealed CD4 $+\mathrm{T}$ lymphocyte predominance, with low percentages of B-lymphocytes and monocytes. Onconeuronal and NMDA-R antibodies were negative in serum and CSF. Serum AQP-4 antibodies were found positive at a titer of 1/40 (indirect immunofluorescence, EUROIMMUN, Medizinische Labordiagnostika, Lübeck, Germany). The pathological study of the ovarian tumor showed a mature cystic teratoma with areas of neural differentiation and AQP-4 expression (Fig. 2). These areas showed inflammatory infiltrates, with intense CD20, CD4 and CD3 lymphocytes, and scattered CD138 positive plasma cells/plasmablasts. The patient was treated with the sequential combination of: 1) a 5-day course of $1 \mathrm{~g} /$ day IV methylprednisolone; 2) seven sessions of plasma exchange; and 3) high dose IV immunoglobulins ( $0.4 \mathrm{~g} / \mathrm{kg} /$ day for 5 days). In addition IV rituximab was administered ( $1 \mathrm{~g} 48 \mathrm{~h}$ before the 4th plasma exchange, $1 \mathrm{~g} 15$ days later). With this strategy the patient progressively improved. After one month of physical therapy she was able to walk with bilateral constant aid, and at three months she was fully ambulatory and able to walk with the occasional use of a cane. Six months later she received two weekly infusions of $1 \mathrm{~g}$ rituximab. At one year follow-up she had no further relapses. The neurological examination was normal except for extensor plantar responses, and repeat brain and spinal MRIs were normal.

\section{Discussion}

The serum and CSF of patients with paraneoplastic myelitis often contain well-characterized onconeuronal antibodies such as anti-Hu, amphiphysin or CRMP5/CV2 (Darnell and Posner, 2011). Symptoms

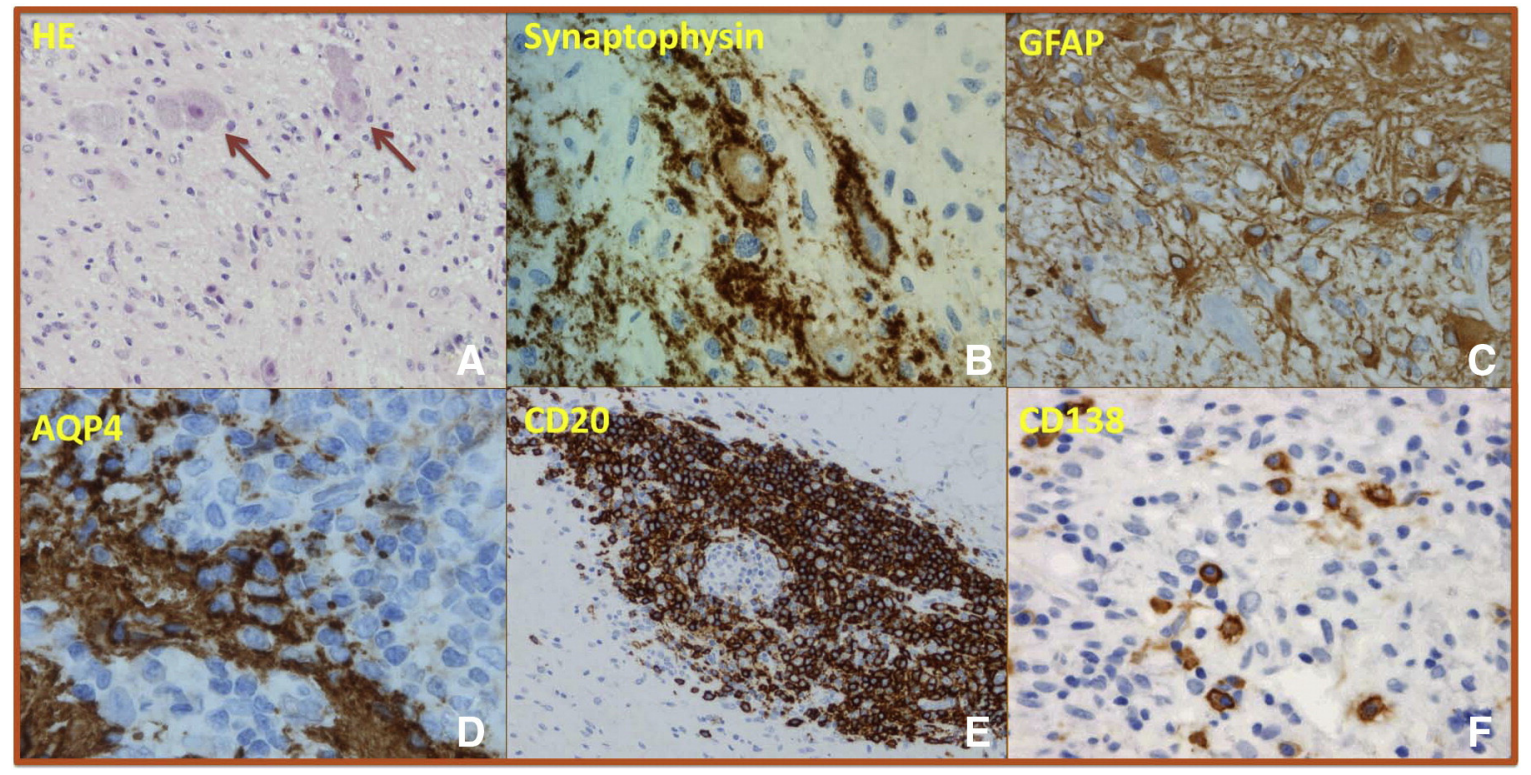

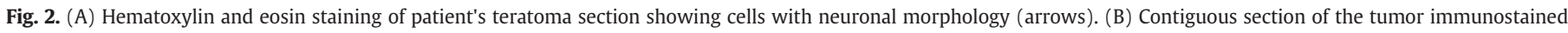

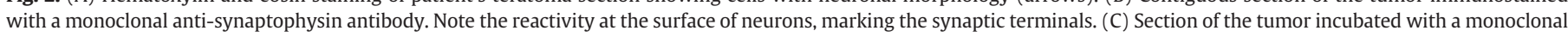

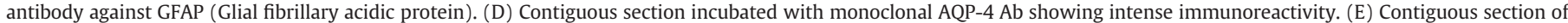

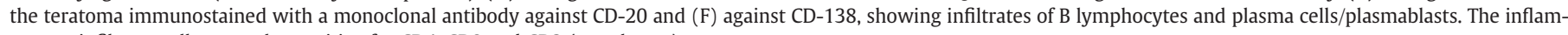
matory infiltrate cells were also positive for CD4, CD8 and CD3 (not shown). 
due to the inflammation of other areas of the central or peripheral nervous system are often seen, and the response to therapies is poor. The tumor expression of the corresponding onconeuronal antigen is a key step in breaking self-tolerance in patients with paraneoplastic neurological syndromes (PNS) associated with onconeuronal antibodies. Malignant cells result in aberrant expression of proteins usually sequestered in the immunoprivileged central nervous system (CNS) thus leading to paraneoplastic autoimmunity. In the patient reported here we demonstrated the expression of AQP4 in areas containing nervous tissue of the ovarian teratoma. These areas were surrounded by intense inflammatory infiltrates. Autoreactive lymphocytes originating in the teratoma could enter the blood stream, cross the blood-brain barrier and cause the neurological disease. Alternatively AQP4 antibodies might be produced peripherally and enter the CNS secondarily (Papadopoulos and Verkman, 2012). In a recent report of a patient with paraneoplastic myelitis, breast cancer and AQP4 antibodies, the tumor was also found to express the AQP4 antigen (Armağan et al., 2012). Because AQP4 is an antigen whose expression is not restricted to the CNS, mechanisms leading to paraneoplastic immunity in patients with AQP4 antibodies probably differ from the classic model proposed for PNS. Intriguingly, patients with AQP4 antibodies have not been reported to develop symptoms due to the damage of peripheral AQP4-expressing organs such as the skeletal muscle or the kidneys.
The incidence of cancer in patients with NMO-spectrum disorders and AQP4 antibodies is unknown, but is likely very low. In a recent series, $5 \%$ of such patients had a history of neoplasm (Pittock and Lennon, 2008). Because only half of these patients developed cancer within 4 years of the neurological symptom onset, the association between both diseases was likely fortuitous for some of them. Future studies should address if there are differences in clinical, immunological and prognostic characteristics between seropositive NMO patients with and without cancer.

\section{References}

Armağan, H., Tüzün, E., İçöz, S., et al., 2012. Long extensive transverse myelitis associated with aquaporin- 4 antibody and breast cancer: favorable response to cancer treatment. J. Spinal Cord Med. 35, 267-269.

Darnell, R.B., Posner, J.B., 2011. Paraneoplastic Syndromes, 1st ed. Oxford University Press, New York.

Flanagan, E.P., McKeon, A., Lennon, V.A., et al., 2011. Paraneoplastic isolated myelopathy: clinical course and neuroimaging clues. Neurology 76, 2089-2095.

Lennon, V.A., Kryzer, T.J., Pittock, S.J., et al., 2005. IgG marker of optic-spinal multiple sclerosis binds to the aquaporin-4 water channel. J. Exp. Med. 202, 473-477.

Mueller, S., Dubal, D.B., Josephson, S.A., 2008. A case of paraneoplastic myelopathy associated with the neuromyelitis optica antibody. Nat. Clin. Pract. Neurol. 4, 284-288.

Papadopoulos, M.C., Verkman, A.S., 2012. Aquaporin 4 and neuromyelitis optica. Lancet Neurol. 11, 535-544.

Pittock, S.J., Lennon, V.A., 2008. Aquaporin-4 autoantibodies in a paraneoplastic context. Arch. Neurol. 65, 629-632. 